\title{
De la détermination des pertes de charge dans l'étranglement des cheminées d'équilibre*
}

\section{The determination of head losses in surge-tank restricted orifices}

\author{
PAR LÉON LEVIN \\ INGÉNIEUR E.I.H., DOCTEUn ¿̀s SCIENCES TECHNIQUES \\ S.F.A.C., USINE DE SAINT-ÉTIENNE
}

\begin{abstract}
L'intérêt et les difficultés d'une évaluation théorique de la perte de charge dans l'étranglement. - Notion $d$ ' "élément créateur de perte de charge » et classification des étranglements d'après le nombre et l'espacement des éléments qui le composent. - Dans bien des cas, la détermination de l'étranglement semble polvoir étre abordée théoriquement avec une bonne approximation (quitte à s'appuyer sur des résultats expérimentaux classiques); dans d'autres cas, le recours au modèle réduit demeure indispensable. - Etude de la courbe perte de charge-débit dans l'étranglement: selon le type de fonctionnement envisagé, son ussimilation is une parabole peut se justifier ou conduire it des erreurs inaceptables.
\end{abstract}

\begin{abstract}
The interest and difficulties of a theoretical evaluation of head loss in throttles - Idea of the " element creating head loss", and classification of throttles according to the number and spacing of the elements which comprise them - In many cases, it seems that near approximation in determination of the throttles can be obtained by theoretical approach (leaving one to apply classic experimental results); in other cases, recourse to the scale model remains indispensable -. Study of the curve for head loss-discharg? in th. throttle: according to the type of operation intended, comparison of the curve to a parabola can either be juslified or can lead to some inacceptable errors.
\end{abstract}

\section{I. - EXPOSE DU PROBLÈME}

On peut admettre actuellement que les méthodes de calcul des oscillations en masse dans les cheminées d'équilibre sont perfectionnées à tel point qu'elles satisfont les besoins des bureaux d'études $\left({ }^{* *}\right)$. Les comparaisons faites en-

(*) Une première ćbautle de cette étude a déjà paru dans la Revue Yougos'ave "Elchtroprivreda 》 (n ${ }^{\circ} \mathrm{i}$, $1952)$.

(“) Les caracteristiques principales des oscillations en masse dans les cheminées cylindriques avec ou sans ctranglement, dans les cheminées à chambres d'expansion et dans les cheminées différentielles sont obtenues aisément à l'aide des abaques de Calame et Gaden [1] et de Frank et Schüllekr. [2].

Pour obtention des caractéristiques secondaires de ces tre les résultats des calculs et les mesures sur les ouvrages en exploitation ont toujours montré une coïncidence très satisfaisante dans les cas où les pertes de charge supposées dans la galerie d'amenée et l'étranglement ont coinncidé avec les pertes de charges réelles dans la galerie et

cheminées ou des caractéristiques principales et secondaires de cheminées plus compliquées ou encore pour la vérification de leur stabilité, on a recours aux intégrations graphiques. Ces méthodes graphiques peuvent être classées en deux groupes :

1. - Celle de Braun-Calame et Gaden-Escande $[3 ; 1 ; 4]$. 2. - Celle de Pressei-Schoklitsch $[5 ; 6 ; 7]$.

Cette dernière a été amenéc par Bovvand et Molbert [8] à une forme très commode dans l'emploi. 
l'étranglement. Autrement dit, les méthodes de calcul graphique et analytique des oscillations en masse ont été sanctionnées par la pratique et le problème principal consiste dans la détermination correcte des pertes dans la galerie d'amenée $\mathrm{P}$ et dans l'étranglement $\mathrm{R}_{0}$. La solution de ce problème rencontre actuellement des difficultés sensibles.

Le premier problème, c'est-à-dire la détermination des pertes dans la galerie d'amenée, se résoud en admettant deux valeurs du coefficient de rugosité de la paroi : une, plus petite, pour le calcul de la montée maximum, et l'autre, plus grande, pour le calcul de la descente maximum (niveau minimum). Nous n'allons pas insister sur ce point déjà traité par ailleurs $[9 ; 10]$, mais nous tenons à rappeler qu'il y a lieu sur ce point d'être très prudent et d'admettre deux valeurs de rugosité pas trop rapprochées. Cette remarque ne doit surtout pas être perdue de vue quand il s'agit d'un enduit au cémentgun sans lissage qui, mettant en ouvre un sable souvent « raide 》 et laissant une surface souvent assez discontinue, admet des coefficients de rugosité $n$ assez élevés $(0,016$ et même 0,017$)$.

Le deuxième problème, e'est-à-dire la détermination des pertes dans l'étranglement, est de nature plus complexe, car il ne s'agit pas d'une " détermination d'un coefficient », mais d'un phénomène hydraulique complexe, où toute analyse hâtive conduirait à des erreurs certai nes. En effet, au pied de la cheminée, se situe un nœud complexe de courants, où le liquide suit non seulement plusieurs appendices (au moins trois), mais encore change alternativement de sens dans ces appendices en régime non permanent. Le phénomène se trouve être difficilement accessible au calcul. Cette circonstance explique la pratique largement répandue dans le monde de l'emploi de modèles réduits pour la détermination des pertes dans l'étranglement; elle explique aussi les opinions discordantes des spécialistes de la question.

Galame et Gaden, Frank et Schuller $[1 ; 2]$ indiquent bien une méthode de calcul des pertes dans l'étranglement, mais déjà Frank ct Schuller (1938) remarquent eux-mêmes que la méthode donne des valeurs exagérées et recommandent, pour y parer, de réaliser l'ètranglement de telle sorte que l'orifice puisse, dans l'exploitation et selon le besoin, être diminué.

JAEGER [11] recommande la méthode de calcul de Frank et Schuller, mais ajoute que des essais sur modèle sont souvent nécessaires.

Escande [12], à la suite d'étude sur modèle et sur l'ouvrage même, calcule l'étranglement conformément à la théorie classique de l'élargissement brusque, c'est-à-dire à l'aide de la formule de Carnot-Borda; cependant, la détermination du coefficient de contraction (qui se trouve dans cette formule) demeure problématique et le Professeur Escandr: recommande la réalisation de l'étranglement au moyen de tôles percées amovibles et interchangeables selon la valeur réelle du coefficient de contraction.

RÉmÉniéras [13] va plus loin et, mème pour un ouvrage essayé sur modèle, projette un étranglement muni de deux volets amovibles de manière à pouvoir effectuer son "réglage », après mise en service de l'usine, en accord avec la perte réelle dans la galerie et en fonction du degré de fidélité de reproduction sur l'ouvrage des détails du dispositif essayé sur modèle.

En présence d'un tel état de la question, nous avons estimé utile - dans l'impossibilité de résoudre le problème dans son ensemble - de préciser ses éléments et de dégager, dans la mesure du possible, quelques critères permettant de classer les étranglements, de dresser des avant-projets pour quelques types relativement simples et de juger de l'opportunité d'étude sur modèle.

\section{II. - ESSAI DE CLASSIFICATION DES ÉTRANGLEMENTS}

\section{A. - Ouvrages longs}

Le caractère et la grandeur de la perte dans l'étranglement sont déterminés par le nombre des éléments qui créent la perte et par la distance séparant ces éléments. Sur le dispositif représenté sur la figure 1 , on distingue trois ćléments créateurs de perte: le branchement, le diaphragme et l'élargissement brusque; la somme de ces trois pertes élémentaires représente la perte de charge dans l'étranglement. Cependant, la grandeur de ces pertes élémentaires, et par conséquent la perte dans l'étranglement, ne nous sont pas connues d'une façon générale.

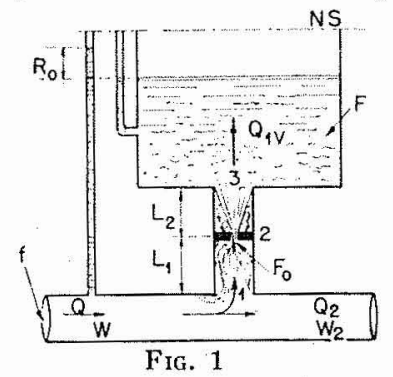


La grandeur de la perte dans l'étranglement put ctre déterminée sur la base de l'hydrauliquo theorique et expérimentale seulement dans un cas particulier, à savoir quand l'élément créawar de perte est suivi d'un conduit cylindrique 16. longueur suffisante pour que la turbulence nocrue par l'obstacle puisse «se calmer 》 et devenir normale eu égard à la vitesse du liquide, $a \mathrm{x}$ dimensions du conduit et à la viscosité du llquide, c'est-à-dire au nombre de Reynouds. Dans ce cas concret, il paraît possible d'addilionner simplement les pertes élémentaires, calculées au moyen des formules de l'hydraulique classique pour chacun des éléments créateurs de perte. Un étranglement satisfaisant à la condilion ci-dessus peut être désigné par ouvrage long.

La méthode de calcul ci-dessus est précisément la méthode de Calame et Gaden, Frank et SchulLER, et JAEGER, mais elle n'est point générale et ne s'applique qu'aux ouvrages longs. L'écart entre le résultat du calcul et la valeur réelle est d'autant plus grand (il peut atteindre 30 et $50 \%$ ) que la distance entre les éléments créateurs de perte est plus petite.

Quel est le critère de qualification de l'ouvrage long? Le cadre de cet article ne nous permet pas de nous étendre sur cette importante question, qui est d'ailleurs traitée dans une autre publication [14], mais nous proposons la formule suivante :

$$
\mathrm{L}_{0}=0,5(\mathrm{D}-d) \sqrt[4]{\mathrm{R}_{e}-10.000}
$$

avec (fig. 2) :

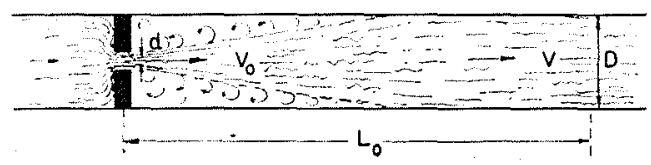

Fig. 2

$\mathrm{L}_{0}=$ longueur minimum d'agitation turbulente accrue;

$\mathrm{D}=$ diamètre du conduit en aval de l'élément-créateur de perte;

$d=$ diamètre de la section contractée;

$\mathrm{R}_{e}=\frac{v \mathrm{D}}{v}$ nombre de RFynolos correspondant à l'écoulement en aval de l'élément créateur de perte.

La formule (1) est basée sur des données expérimentales correspondant à $\mathrm{R}_{e}=28.000$ à 500.000 et $\mathrm{D} / d=1,25$ à 3,00 .

\section{B. - Ouvrages courts \\ a) Ouvrages courts simples.}

Que se passe-t-il dans les étranglements où la distance entre les éléments créateurs de perte est inférieure à la valeur obtenue par la formule (1)? Avant d'aborder ce problème complexe, nous nous proposons de résoudre le probleme simple posé par un étranglement composé d'un

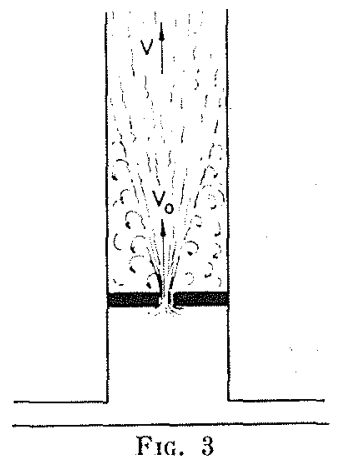

seul élément créateur de perte, par exemple un diaphragme monté dans la cheminée d'équilibre même [12]. Ce problème relève du domaine de l'hydraulique classique et se résoud au moyen de la formule théorique de Carnot-Borda :

$$
\mathrm{H}=\frac{\left(v_{0}-v\right)^{2}}{2 g}
$$

avec (fig. 2 et 3 ):

$v_{0}=$ vitesse dans la section contractée;

$v=$ vitesse moyenne dans le conduit aval;

$\mathrm{H}=$ perte de charge dans l'étranglement.

La formule (2) a été vérifiée expérimentalement maintes fois (en régime turbulent évidemment) au moyen des mesures des grandeurs $v_{0}, v$ et des pressions dans la section contractée et dans la section éloignée de cette dernière d'une longueur $\mathrm{L}_{0}$. Cependant, dans les cheminées d'équilibre, la grandeur $v_{0}$ ne nous est pas toujours connue du fait de l'incertitude quant à la valeur du coefficient de contraction $m$ de la formule:

$$
v_{0}=\frac{\mathrm{Q}}{m \mathrm{~F}_{0}}
$$

avec :

$$
\begin{aligned}
& \mathrm{Q}=\text { débit traversant l'étranglement; } \\
& \mathrm{F}_{0}=\text { section droite de l'ouverture de l'étran. } \\
& \text { glement. }
\end{aligned}
$$

La valeur du coefficient de contraction $m$ nous est fournie par l'expérience. Pour un très grand nombre d'éléments créateurs de perte (branchement, coude, diaphragme, ajutage, etc.), la valeur de ce coefficient est connue depuis longtemps et peut être trouvée dans différents traités d'hydraulique $[14,15,16,17]$ et autres. Pour 
un ouvrage important, dont la forme se différencie beaucoup des formes d'ouvrages déjà essayés, on pourra avoir recours aux essais sur modèle réduit, qui fournissent la valeur du coefficient de contraction avec une grande précision.

Un étranglement, composé d'un seul élément créateur de perte, est dénommé ouvrage court simple.

\section{b) OUvrages courts complexes.}

Nous pouvons maintenant nous tourner vers le problème posé par la présence dans un étranglement de plusieurs éléments-créateurs de perte. Le cas d'ouvrages longs a été déjà traité. Nous y ajouterons seulement que la détermination de la perte de charge dans l'étranglement consiste à appliquer la formule (2) autant de fois qu'il y a d'éléments et à additionner les résultats correspondants. Par exemple, pour l'étranglement représenté sur la figure 1 , au cas où $L_{1} \geqslant L_{01}$ et $\mathrm{L}_{2} \Rightarrow \mathrm{L}_{02}$, on emploie la formule (2) trois fois : pour le branchement, pour le diaphragme et pour l'élargissement brusque $\left(^{*}\right)$; la somme des trois résultats fournit la perte de charge dans l'étranglement.

Quelle est la perte de charge dans l'étranglement quand les éléments créateurs de perte sont rapprochés à tel point que certains d'entre eux n'influent point sur la section contractée? En se reportant à la figure 1 , on peut intuitivement concevoir qu'au cas où les longueurs $L_{1}$ et $\mathrm{L}_{2}$ sont très courtes, il ne se formera qu'une seule contraction, indépendamment $d u$ fait de la présence de trois éléments de perte. Dans ce cas on peut dire que les contractions des trois éléments se confondent en une contraction, dite contraction complexe, et un tel étranglement pourrait être dénommé oubrage court complexe.

Théoriquement, le problème est dès lors clair : étant donné qu'il n'y a qu'une contraction, la perte de charge est fournie par la formule (2), mais pratiquement le problème n'est pas résolu, car la valeur du coefficient de contraction $m$ de la formule (3) n'est pas connue. A la lumière de ce fait, on peut dire que le problème des ouvrages courts complexes est identique à celui des ounrages courts simples; sous la seule réserve que si, pour ces derniers, la valeur du coefficient de

(*) La forme des formules de l'hydraulique classique pour les pertes de'charge singulières (branchement par exemple) peut différer de la formule (2), mais leur sens est précisément celui que fait clairement ressortir la formule de Cannor-Bonda : toute perte de charge singulière (sauf celle des courbes) n'est antre qu'une perte par élargissement brusque, car le « rétrécissement brusque » ne proonque presque aucune perte. Seule la valeur du coefficient de contraction differe selon la forme de l'ouvrage. contraction est déterminée depuis longtemps, cette tâche reste à accomplir pour les premiers.

Différents laboratoires dans le monde ont déterminé sur modèle les coefficients de contraction de différents ouvrages courts complexes [12, 13, 18]. Le Laboratoire d'Hydraulique d'Avala (Yougoslavie) a étudié expérimentalement les étranglements, qui sont des ouvrages courts complexes, des cheminées des centrales hydro-électriques de Jajce II, de Jablanica I, de Jablanica II, de Glava Zeta [14]. Ces données expérimentales jouent précisément, pour les ouvrages courts complexes, le même rôle que la riche documentation expérimentale acquise durant la deuxième moitié du $\mathrm{xr}^{\circ}$ siècle et le début du $\mathrm{xx}^{\prime \prime}$ siècle pour les ouvrages courts simples. La multiplication des études sur modèle et l'échange entre les laboratoires des résultats obtenus auront pour conséquence que les coefficients de contraction de nombreux ouvrages courts complexes seront tout aussi connus que ceux correspondant aux ouvrages courts simples.

Etant donné que l'ingénieur dispose souvent d'assez de liberté dans le tracé de l'étranglement, il tendra vers des formes déjà étudiées sur modèle, ce qui lui permettra d'éviter une étude expérimentale spéciale et de raccourcir les délais d'élaboration du projet.

Pour une détermination approximative du coefficient d'un ouvrage court complexe, nous proposons la règie empirique suivante (vérifiéce sur plusieurs modèles à 5 à $10 \%$ près) : en présence d'une contraction complexe, on adopte, pour l'ouvrage, le coefficient de contraction de celui des éléments créateurs de perte qui, dans l'hypothèse de marche sur un ouvrage long, aurait donné la plus grande perte. Par exemple, si l'étranglement représenté sur la figure 1 accuse, dans l'hypothèse, qu'il soit ouvrage long, les coefficients de contraction suivants (par rapport au tube vertical) :

$$
\begin{array}{ll}
\text { Pour le branchement........ } & m=0,58 \\
\text { Pour le diaphragme......... } & m=0,42 \\
\text { Pour l'élargissement brusque.. } & m=1,00
\end{array}
$$

on adoptera pour cet étranglement, quand il est ouvage court complexe, $m=0,42$.

Reste à définir un critère des ouvrages courts complexes: quel est l'écart maximum $\mathrm{L}_{0}^{\prime}$ entre deux éléments créateurs de perte pour que l'étranglement puisse être considéré comme ouvrage court complexe? Il n'est pas aisé de donner actuellement une réponse précise à cette question. Cependant, il est'connu que la contraction a lieu pour les orifices circulaires à une distance égale à leur rayon $d / 2$; la section contractée se maintient quasi-cylindrique sur un 
Gourt parcours pour diverger ensuite, si l'écouInmenl a lieu dans l'eau, sous un angle au somwet de $20^{\circ}$ environ.

Gelte propriété peut être utilisée pour se donwh un critere des ouvrages courts complexes of nous proposons la définition suivante (en généralisant le résultat des orifices circulaires anx urilices de loule forme et en posant $d=4 R$ ) :

Un étranglement est considéré comme ouvrage court complexe quand la distance entre les éléments créteurs de perte ne dépasse pas la valeur:

$$
\mathrm{L}_{0}^{\prime}=3 \text { à } 4 \mathrm{R}
$$

avec $\mathrm{R}$ = rayon hydraulique de la section qui a provoqué la contraction.

It faut souligner que la majorité des étranglements projetés satisfont bien à la formule (4).

\section{C. - Ouvrages hydrauliquement indéterminés}

Restent les étranglements qui ne sont ni ouvrages longs, ni ouvrages courts, e'est-à-dire ceux où la distance $\mathrm{L}$ entre les éléments créateurs de perte est :

$$
\mathrm{L}_{0}^{\prime}<\mathrm{L}<\mathrm{L}_{0}
$$

L'étranglement ainsi conçu est un onvrage $h y$ drauliquement indéterminé et la perte de charge qu'il provoque ne pourrait, sur la base de nos connaissances actuelles de l'hydraulique, être calculée. Souvent, on peut d'ailleurs éviter une telle conception de l'étranglement et traiter le dispositif projeté soit en ouvrage long, soit en ouvrage court. Au cas où aucune de ces dispositions ne peut ètre adoptée, une étude sur mo. dèle devient nécessaire, sinon indispensable.

\section{III. - COURBES DES PERTES DE CHARGE DANS L'ÉTRANGLEMENT}

Il importe avant tout de préciser que « la perte de charge dans l'étranglement »n'est pas simplement une perte de charge, mais contient aussi des termes de hauteurs représentatives de vitesse. Les expressions pour $R_{0}$ et $R_{0}^{\prime}$ sont $[14$; voir aussi 2] :

Pour: la montée (fig. 1):

$$
\mathrm{R}_{0}=\mathbf{H}-\frac{w^{2}}{2 g}+\frac{v^{2}}{2 g}+\zeta_{b} \frac{w^{2}}{2 g}
$$

Pour $Q_{1}=0$, le coefficient de perte dans le branchement $\zeta_{b}$ prend la valeur $\zeta_{b_{0}}$ qui est une grandeur positive $[19,20$ et 21$]$.

Pour la descente (fig. 4):

$$
\mathrm{R}_{0}^{\prime}=\mathrm{H}^{\prime}+\frac{w^{2}}{2 g}-\frac{v^{2}}{2 g}+\zeta^{\prime}{ }^{\prime} \frac{w^{2}}{2 g}
$$

Pour $Q_{1}=0$, le coefficient de perte dans le branchement (sens descente) $\zeta^{\prime}{ }_{b}$ prend la valeur $\zeta_{b 0}^{\prime}$ qui est une grandeur négative $[19,20$ et 21].

La formule (5) donne la valeur $R_{0}$ par rapport à la section du tunnel d'amenée située en amont du branchement, tandis que l'équation (6) donne la valeur $R_{0}^{\prime}$ par rapport à la section du tumnel située en aval du branchement. On peut, d'ailleurs, établir aussi une relation pour $R_{0}^{\prime}$ (quoique un peu plus compliquée) par rapport à la section en amont du branchement, relation qui serait tout aussi valable que l'équation (6), à condition toutefois d'accompagner chacune de ces valeurs $R_{0}^{\prime}$ par la perte de charge corres- pondante P dans le tunnel d'amenée; autrement dit, dans le cas du calcul de $\mathrm{R}_{0}^{\prime}$ par rapport à la section aval, on comprendra dans la perte de charge $\mathrm{P}$ la perte due au branchement au pied de la cheminée [14].

Dans l'exécution de l'intégration graphique, on se sert des courbes $R_{0}$ et $R_{0}^{\prime}$ en fonction du débit $Q_{1}$.

a) Montée du plan d'eau dans la cheminée (fig. 1).

L'équation (5) prend pour les ouvrages courts (par emploi de l'équation 2) l'aspect suivant :

$$
\mathrm{R}_{0}=\frac{\left(w_{0}-v\right)^{2}}{2 g}-\frac{w^{2}}{2 g}+\frac{v^{2}}{2 g}+\zeta_{b} \frac{w^{2}}{2 g}\left(5^{\prime}\right)
$$

Etant donné que l'on admet généralement pour le calcul de la montée maximum une fermeture totale des turbines (de $100 \%$ à 0 ), on peut cicrire $Q=Q_{1}$ et l'équation $\left(5^{\prime}\right)$ devient :

$\mathrm{R}_{0}=\frac{\left(\frac{1}{m \mathrm{~F}_{11}}-\frac{1}{\mathrm{~F}}\right)^{2}-\frac{1-\zeta_{b}}{f^{2}}+\frac{1}{\mathrm{~F}^{2}}}{2 g} \mathrm{Q}_{1}{ }^{2}=\mathrm{K} \mathrm{Q}_{1}{ }^{2}$

expression où $\mathrm{K}$ est une constante et, par conséquent, $\mathrm{R}_{0}$ est une parabóle. (Théoriquement, $K$ est bien fonction du niveau dans la cheminée d'équilibre, mais pour la plupart des ouvrages, cette dépendance est pratiquement négligeable). 
Pour un ouvrage long, l'équation (5) devient : $\mathrm{R}_{0}=\frac{\Sigma\left(\nu_{0 i}-v_{i}\right)^{2}}{2 g}-\frac{\left(1-\zeta_{b}\right) w^{2}}{2 g}+\frac{\eta^{2}}{2 g}=\mathrm{K} Q_{1}{ }^{2}$ et la seule différence par rapport aux ouvrages courts consistera dans le fait que dans l'expression correspondant aux ouvrages longs figure la somme :

$$
\Sigma\left[\frac{1}{\left(m \mathrm{~F}_{0}\right)_{i}}-\frac{1}{\mathrm{~F}_{i}}\right]^{2}
$$

a la place d'un terme unique.

\section{b) Descente du plan d'eau (fig. 4).}

L'équation (6) prend, pour les ouvrages courts, l'aspect suivant :

$$
\mathrm{R}_{0}^{\prime}=\frac{\left(p_{0}-w\right)^{2}+w^{2}-v^{2}+\zeta_{b}^{\prime} w^{2}}{2 g}
$$

Cependant, on calcule rarement une cheminée pour une ouverture totale des turbines, ce qui a

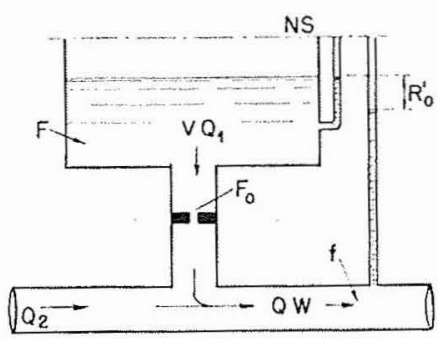

FiG. 4

pour conséquence de compliquer le calcul.

L'équation $\left(6^{\prime}\right)$ prend - du fait de la nonvalidité de la formule CARNOT-BORDA aux branchements - un caractère empirique; elle s'écrit :

$$
\begin{gathered}
\mathrm{R}_{0}{ }^{1}=\frac{1}{2 g}\left[\left(\frac{1}{m \mathrm{~F}_{0}}-\frac{\mathrm{Q}}{\mathrm{Q}_{1}} \cdot \frac{1}{f}\right)^{2}\right. \\
\left.+\left(\frac{\mathrm{Q}}{\mathrm{Q}_{1}}\right)^{2}\left(1+{\zeta_{b}}_{b}\right) \cdot \frac{1}{f^{2}}-\frac{1}{\mathrm{~F}^{2}}\right] \mathrm{Q}_{1}{ }^{2}=\mathrm{K}^{\prime} \mathrm{Q}_{1}{ }^{2}
\end{gathered}
$$

L'équation (8), valable pour une ouvertiure partielle, se distingue essentiellement de l'équation (7), valable pour une fermeture totale, par le fait que le facteur $\mathrm{K}^{\prime}$ est très influencé par le rapport des débits $\mathrm{Q}_{1} / \mathrm{Q}$, et que, par conséquent, la courbe $\mathrm{R}_{0}{ }^{1}$ en fonction du débit $\mathrm{Q}_{1}$ n'est pas une parabole.

Cette dépendằnce se fait particulièrement sen- tir sur les ouvrages courts avec prépondérance de l'élément branchement. Pour cette catégorie d'ouvrages, il est possible d'effectuer un calcul de première approximation sur la base des études expérimentales très sérieuses effectuées en 1926-31 à l'Institut d'Hydraulique de Munich

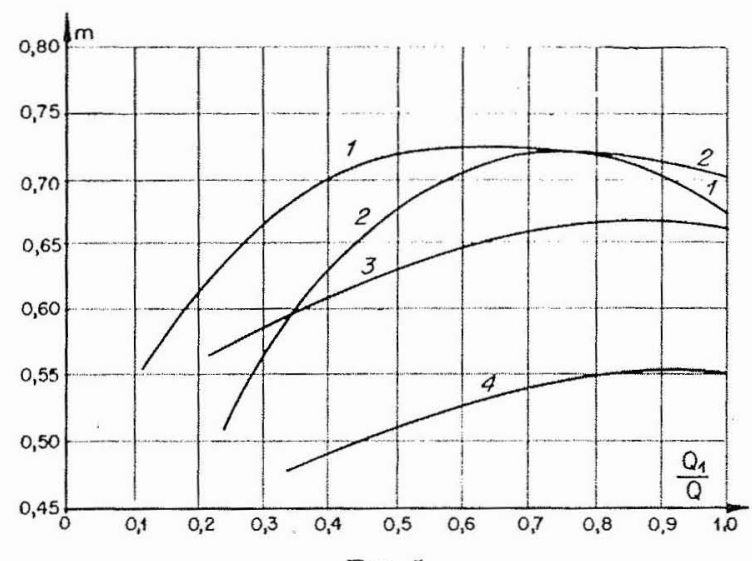

Fig. 5

sous la direction de Thoma. Dans les publications de cet Institut $[19,20$ et 21], on trouve les valeurs du coefficient $m$ pour de très nombreuses dispositions du branchement.

Nous reproduisons sur la figure 5, à titre

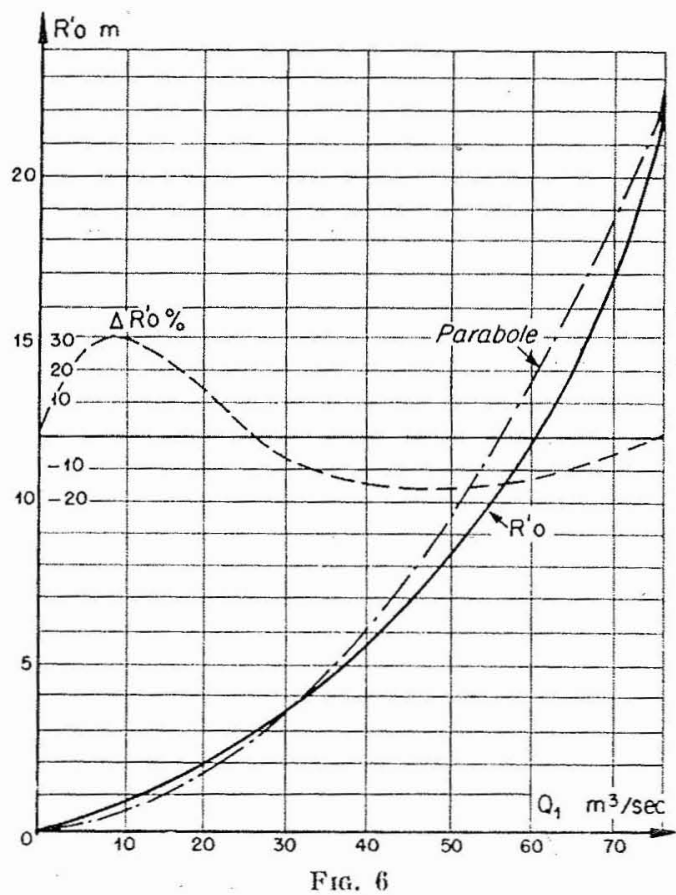

d'exemple, quatre courbes du coefficient de contraction $m$ en fonction du rapport des débits $Q_{1} / Q$, obtenues sur modèles réduits d'ouvrages courts. On peut constater à première vue que la variation du coefficient n'est pas négligeable. 
Whus donnons sur la figure 6 , pour la courbe 2 do lo figure 5, la courbe réelle des pertes de churge dans l'étranglement et la parabole qui fonne pour les points extrêmes $\left(Q_{1}=0\right.$ et 6. Q) les mèmes valeurs que la courbe réelle. on a tracé également l'écart entre les deux courbes. On voit, daprès la figure 6 , que les écarts extremes ont atteint $-17 \%$ et $+30 \%$. De tels venrts sur la perte dans l'étranglement entraîtent des écarts inadmissibles dans le calcul de foscillation en masse dans la cheminée et surtout dans celui de la stabilité.

Pour les ouvrages longs, l'équation $\left(6^{\prime}\right)$ ne diffère de celle concernant les ouvrages courts que par l'intervention de la somme :

$$
\Sigma\left(v_{0 i}-w_{i}\right)^{2}
$$

à la place d'un terme unique. Cependant, il faut remarquer que, dans ce cas, la dépendance de $\mathrm{K}^{\prime}$ vis-a-vis du rapport des débits $\mathrm{Q}_{1} / \mathrm{Q}$ est nettement plus atténucé que pour les ouvrages courts; et souvent elle est négligeable.

Il est à peine utile d'ajouter que, en cas de calcul d'une fermeture partielle des turbines, on aboutit aux mêmes conclusions générales que l'on vient d'établir pour l'ouverture partielle.

\section{IV. - RESUMÉ ET CONCLUSIONS}

1. - Les méthodes de calcul actuelles des oscillations en masse dans les cheminées d'équilibre satisfont pleinement les bureaux d'études et la seule difficulté dans l'application se trouve être la détermination des pertes de charge dans le tunnel d'amenée et dans l'étranglement.

2. - On ne dispose pas actuellement d'une méthode de calcul sùre de la perte de charge dans l'étranglement, ce qui explique l'emploi fréquent dans ce but du modèle réduit.

3. - Le nombre d'éléments créaleur: de perte et la distance entre ellx déterminent la perte de charge totale dans l'étranglement. Quand la distance entre ces éléments est égale ou supérieure à $\mathrm{L}_{0}$ de l'équation (1), le dispositif est un ouvrage long et la perte totale est obtenue simplement en additionnant les pertes élémentuires, calculées selon les formules classiques de l'hydraulique.

4. - Quand l'étranglement est constitué d'un seul élément, le dispositit est un ouvrage. court simple; la perte de charge dans l'étranglement est fournie par la formule théorique de Carnot-Borda (équation 2), et le coefficient de contraction $m$ de l'équation (3) est déterminé expérimentalement.

Les coefficients de contraction de la plupart des ouvrages courts simples sont connus depuis longtemps et peuvent etre trouvés dans divers traités d'hydraulique $[14,15,16,17$,etc. $]$.

5. - Quand l'étranglement est constitué de plu- sieurs éléments séparés par des intervalles ne dépassant pas la valeur $L_{0}^{\prime}$ de l'équation (4), toutes les contractions élémentaires fusionnent en une seale et le dispositif est un ouvrage court complexe. La perte totale est encore foumie par la formule (2); le coefficient de contraction est déterminé expérimentalement $[12,13,14$, 18 ].

Il importe d'adopter pour les étranglements des ourrages courts complexes aes formes, dont le coefficient de contraction est déjà connu; dans le cas contraire, une étude sur modèle réduit est nécessaire.

Pour l'évaluation de la valeur du coefficient, en preinière approximation (à $10 \%$ près environ), nous proposons la règle empirique suivante : admeltre pour louvrage court complexe le coefficient de contraction de celui des éléments créateurs de perte qui, dans l'hypothèse de marche sur un ouvrage long, donnerait la perie maximum.

6. - Au cas où la distance entre les éléments est comprise entre les valeur's $L_{0}$ (équation 1) et $L_{0}^{\prime}$ (équation 4), l'étranglement est hydrauliquement indéterminé et l'étude sur modèle est indispensable.

7. - Les expressions des «pertes de charges » dans l'étranglement $R_{0}$ et $R_{0}^{\prime}$ comprennent, à côté des termes principaux de la perte de charge, des termes de hauteurs de vitesse (équations 5 et 6 ).

8. - La courbe $R_{0}$, en fonction du débit à travers l'étranglement $Q_{1}$, est, pour une fermeture totale des turbines, une parabole. 
9. - La courbe $\mathrm{R}_{0}^{\prime}$, pour une ouverture partielle des turbines, est fonction du rapport des débits $Q_{1} / Q$ (équation 8). Elle s'éloigne sensiblement de la parabole ( 30 \% et plus) sur les ouvrages courts complexes (voir exemple sur fig. 6), ou le coefficienl de contraction $m$ est forlement dépendant du rapport Q, $/ Q$. L'emploi de la parabole mènerait dans ces cas à des erreurs appréciables dans les calculs d'oscillations en masse et de slabilité.

\section{Bibliographie}

1 Calame et Gaden. - Theorie des chambres d'équilibre. Dunod, 1926.

2] Fraxk und ScHürLER. - Schuingungen in den $Z$ uleitungs und Ableitungskänalen von Wasserkaftanlagen. Springer, 1938.

[3] Braun. - Ueber graphische Behandlung des Wasserschloss probleme. Schweiz. Bauztg., Bd 77, 1921.

[4] L. Escande. - Méthodes nouvelles pour le calcul des chambres d'équilibre. Dunod, 1950.

[5] Presser. - Beilrag zur Bemezzung des Inhalles bon Wasserschlössern. Sehweiz. Bauztg., Bd 53, 1909 .

(i) Schokntwch. - Schweizerische Banzeilung. Band 79, 1923 .

[7] Schokutsch. - Der Wasserbau. Springer, 1950.

[8] Bovvand et Monmmt. - Mélhode graphique ponr le calcul des cheminées d'équilibre. "La Houille Blanche $\gg, n^{\circ} 9,1950$ et $n^{\circ} 3,1951$.

[9] V. Jevdievic. - O odredjiuanju qubilaka u prigusenju lod odostana. "EIcktroprivreda 》, br. 2, 1952.

[10] L. Leves. - Doprinos o odredjinanju gnbilaka $u$ stolni prilikom projektonanja podostana. "Slektropriveda $\gg, \mathrm{br} .3,1952$.

[1] Ch. JaEcen. - Technische Hydraulik. Birkhäuser, 1949 .
0127 L. Escanos - Recherches hioriques at crperimenlales sur les oscillations dans les chambres d'equilibre. Direction de l'Aeronatique, 1943.

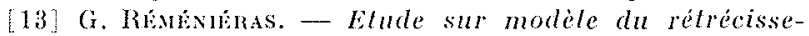
ment de la cheminèe d'équilibre de Saint-Geniez. Arehives de la Sociéte Hydrotechnique de France. 1944 (inédit).

[14] L. Levix. - Odredjivanje gubitaka u prigusenju $u$ vezi proracuna bodostana (en impression).

[15] G. RovTs. - Conrs d'hydraulique industrielle. Paris, 1925.

[16] WeYmavch-Stroned. - Hydraulisches. Rechnen, 1930 .

[17] C.V. Davis. - Handbook of Applied Hydratics New-York, 1949.

(18) L. Escande. - Elude sur modèle réduit de l'ajuluge dissymetrique constifuant l'etranglement at lu base des cheminées d'équilibre, 1949 (inédit).

[19] G. VoGes. - Untersuchungen übr den Verlust in rechtwinklingen rohrverzueigungen. -... Mitteil. d. Hydr. Inst, d. Techn. Hochsch, Mänchen, Heft 1,1926 und 2,1929 .

20] F. Petermanx. - Der Verlust in Schiefminkeligen Rohrverzweigungen. M.H.I.T.H.M. Heft 3, 1929.

21 E. Kowe. - Beitrage zur lionntniss der Hydraulischer Verluste in Abbzweigetürlen. M.H.I.T.H.M.. Heft 4,1931 .

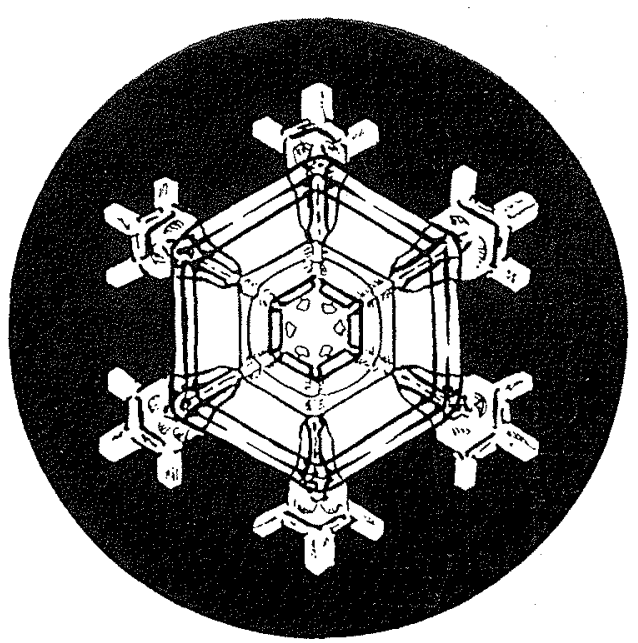

